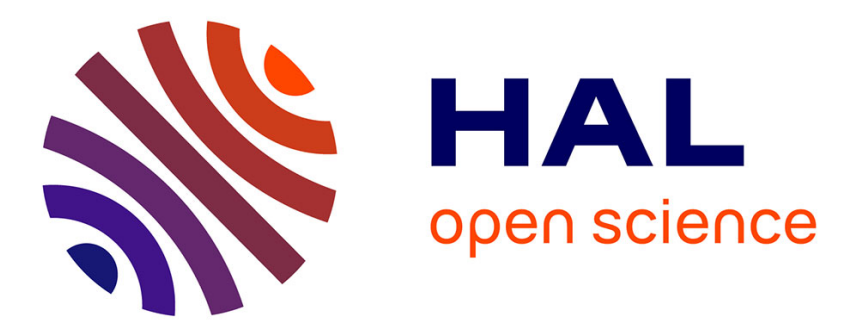

\title{
Experimental and theoretical investigation of the heating of combusting droplets in a linear stream
}

Guillaume Castanet, Pascal Lavieille, Michel Lebouché, Fabrice Lemoine

\section{To cite this version:}

Guillaume Castanet, Pascal Lavieille, Michel Lebouché, Fabrice Lemoine. Experimental and theoretical investigation of the heating of combusting droplets in a linear stream. Combustion Science and Technology, 2005, 177 (12), pp.2395 - 2422. 10.1080/00102200500241206 . hal-01570427

\section{HAL Id: hal-01570427 \\ https://hal.univ-lorraine.fr/hal-01570427}

Submitted on 30 Jul 2017

HAL is a multi-disciplinary open access archive for the deposit and dissemination of scientific research documents, whether they are published or not. The documents may come from teaching and research institutions in France or abroad, or from public or private research centers.
L'archive ouverte pluridisciplinaire HAL, est destinée au dépôt et à la diffusion de documents scientifiques de niveau recherche, publiés ou non, émanant des établissements d'enseignement et de recherche français ou étrangers, des laboratoires publics ou privés. 


\title{
EXPERIMENTAL AND THEORETICAL INVESTIGATION OF THE HEATING OF COMBUSTING DROPLETS IN A LINEAR STREAM
}

\author{
G. CASTANET \\ P. LAVIEILLE \\ M. LEBOUCHÉ \\ F. LEMOINE \\ LEMTA, CNRS, Vandœuvre-lès-Nancy cedex, France
}

Combusting ethanol droplets in linear stream are investigated by two colors laser induced fluorescence. The technique enables us to follow the transient mean temperature evolution of the combusting droplets. A database, including a wide range of aerothermal injection parameters such as injection velocity, droplet diameter and inter-droplet distance (or non-dimensional distance parameter) is provided. A calculation based on the heat diffusion equation resolution, with a constant temperature fixed at the measured equilibrium temperature has been implemented. Both heat conduction and heat advection by the droplet internal motions have been taken into account, the inside droplet velocity field being modeled by the Hill vortex solution. The influence of the different injection parameters are discussed and the results are compared with the models usually available in the literature. The measurement relative to the droplets' transient heating enable to determine the Hill vortex intensity related to the maximum velocity at the droplet.

Keywords: laser-induced fluorescence, droplet, monodisperse stream, combustion

This work is supported by the European Community in the framework of the MUSCLES contract, Growth project GRD1-2001-40198. 


\section{INTRODUCTION}

Liquid fuels are commonly used in propulsion devices such as aeroengines. The liquid fuel is introduced into the combustion chamber by high pressure atomizers as a liquid spray. The optimization of the fuel consumption and the reduction of pollutant formation require a sharp understanding of the spray behavior in the combustion chamber. Fuel droplets range typically from a few $\mu \mathrm{m}$ to a few $100 \mu \mathrm{m}$ in diameter, with velocities of a few $10 \mathrm{~m} / \mathrm{s}$. After being atomized, the droplets enter in a high temperature environment; thus, the droplets are heated, evaporate and the fuel vapor burns and delivers the energy for propulsion. In usual turbojet combustors, the duration of the heating phase is not negligible compared to the total transit time of the droplet in the combustion chamber. Consequently, physical heating models of droplets must be implemented in the predictive calculation tools. Due to the complexity of the experiments on full scale combustors, simple configurations are used in the field of the improvement of the physical models. Monodisperse droplet arrays or lines are examples of laboratory experiments which have been widely used in either experimental (Mulholland et al., 1988; Silverman and Dunn-Rankin, 1994) and theoretical studies (Chiang and Sirignano, 1993). Prior numerical studies have shown that the heat transfer inside a droplet was the results of combined conduction and advection due to the formation of internal vortices, caused by the friction phenomena between the liquid droplet surface and the external air flow (Abramzon and Sirignano, 1989; Chiang et al., 1992; Sazhin et al., 2002). Jin and Borman (1985) were the first to introduce the concept of effective conductivity, which corresponds to a heat conductivity modified by the inside droplet convective motions.

The understanding of the heating process of a fuel droplet entering in a hot gas environment requires the measurement of the droplet mean temperature, i.e., the temperature spatially averaged on the droplet entire volume, which is related to the droplet enthalpy. The complete resolution of the heat convection/diffusion equation, assuming a velocity field given by the Hill vortex solution, and the effective conductivity model will be discussed in the light of experimental data collected on a combusting monodisperse ethanol droplet stream. The two colors laser-induced fluorescence technique, using one single fluorescent tracer and two detection spectral bands, has been developed by Lavieille et al. $(2001 \mathrm{a}, 2002)$ in order to measure the mean droplet 
temperature in a monodisperse droplet stream in simple evaporation or in combustion regime.

The main advantages of the two colors laser-induced fluorescence technique are the total independence of the measurement on the measuring volume, i.e., the fraction of the droplet illuminated by the laser excitation, the local laser intensity and the tracer concentration. The complete heating phase of the droplet will be studied, starting at the ignition up to the position where the droplets have reached there equilibrium temperature, corresponding to the wet-bulb temperature. The influence of several parameters such as the droplet diameter, the injection velocity or the distance parameter, characterizing the droplet to droplet aerodynamical interactions, defined by the ratio between the droplet spacing and droplet diameter, will be considered in this work.

\section{PRINCIPLES OF TWO COLORS LASER-INDUCED FLUORESCENCE APPLIED TO THE MEAN DROPLET TEMPERATURE MEASUREMENT}

\section{Principles}

The main outlines of the two colors laser-induced fluorescence technique are detailed in this section. Further technical details can be found in a previous paper (Lavieille et al., 2001a). The fuel, ethanol here, is previously seeded with a low concentration (a few $\mathrm{mg} / \mathrm{l}$ ) of rhodamine $\mathrm{B}$. Rhodamine B is an organic dye usually used as fluorescent temperature sensor. Furthermore, the fluorescence of rhodamine B can be easily induced by the green line $(\lambda=514.5 \mathrm{~nm})$ of the argon ion laser and is a suitable candidate to be a temperature tracer (Copetta and Rogers, 1998). The rhodamine B fluorescence spectrum is broadband, and it has been shown that its temperature sensitivity was strongly depending on the wavelength (Lavieille et al., 2001a). The fluorescence intensity expression, integrated on a given spectral band of detection, as a function of the different physical and optical parameters is given by (Castanet et al., 2003; Lavielle et al., 2001a; Lemoine et al., 1999):

$$
I_{f i}=K_{\text {opti }} K_{\text {speci }} V_{c} I_{0} C e^{\frac{a_{i}}{T}+\frac{b_{i}}{T^{2}}}
$$

where $i$ denotes the given detection spectral band, $K_{\text {opti }}$ is an optical constant, $K_{\text {speci }}$ is a constant depending solely on the spectroscopic properties of the fluorescent tracer in its environment (i.e., the fuel), $I_{0}$ the laser 
excitation intensity, $C$ the molecular tracer concentration, $T$ the absolute temperature, $V_{c}$ is the fluorescence photons collection volume. The product $C \cdot V_{c}$ of the collection volume by the tracer molecular concentration is in fact related to the number of fluorescence photons emitted by the rhodamine $\mathrm{B}$ molecules excited by the laser radiation and reaching the photodetector surface. This parameter is strongly related to the droplet size and to the probe volume dimensions. The parameters $a_{i}$ and $b_{i}$ are the temperature sensitivity coefficients for the spectral band i. In order to measure properly the temperature of a moving and combusting droplet, the influence of the parameters $C \cdot V_{c}$ and $I_{0}$ must be removed. In combustion situations, the dye concentration is also likely to vary and the collection volume is constantly changing as the droplet crosses the probe volume. Furthermore, the intensity distribution of the laser excitation within the droplet depends on the position of the droplet due to the lensing effect of the droplet interface. In order to get rid of these problems, the fluorescence intensity is detected on two spectral bands for which the temperature sensitivity is highly different. The selection of these spectral band is optimized by an initial study of the fluorescence spectrum against temperature (Lavieille et al., 2001a). The fluorescence ratio between both fluorescence intensities collected on both optimal spectral bands is given by:

$$
R_{f}=\frac{I_{f 1}}{I_{f 2}}=\frac{K_{\text {opt } 1}}{K_{\text {opt } 2}} \frac{K_{\text {spec } 1}}{K_{\text {spec } 2}} e^{\frac{a_{1}-a_{2}}{T}+\frac{b_{1}-b_{2}}{T^{2}}}
$$

This ratio is totally independent of the dimensions of the intersection between the droplet, the laser excitation volume and the photon collection volume. The influence of the local laser excitation intensity and tracer concentration are also totally eliminated. The use of a single reference point where the temperature is known allows the elimination of the optical and spectroscopic constants. An initial calibration, performed under static conditions, consisting in progressively heating the fuel in a vessel, enables the determination in one test of the coefficient set $\left(a_{1}-a_{2}\right)$ and $\left(b_{1}-b_{2}\right)$. The fuel (ethanol) for the combustion tests has been prepared as recommended by Lavieille et al. (2001b), with a dye concentration $\mathrm{C}=2 \cdot 5 \cdot 10^{-6} \mathrm{~mol} / 1$ (rhodamine $\mathrm{B}$ ) and a large excess of sodium hydroxide $(\mathrm{NaOH})$ in order to stabilize the rhodamine $\mathrm{B}$ in a basic medium. This prevents the acidification process of the liquid ethanol during the combustion, since the rhodamine B fluorescence spectrum evolves with the $\mathrm{pH}$ value in an acid medium. By this way, 
the fluorescence ratio becomes insensitive to any changes in the composition of the liquid phase. For this fuel and for the present optical set-up, described next, the temperature sensitivity coefficients are $\left(a_{1}-a_{2}\right)=$ $-4462.9 \mathrm{~K}$ and $\left(b_{1}-b_{2}\right)=5.524 \cdot 10^{5} \mathrm{~K}^{2}$. The resulting temperature sensitivity is about $2 \% / \mathrm{K}$.

\section{Optical Setup}

The optical setup can be divided into two parts : the emission optics and the reception set-up which includes the collection optics and the set-up which divides the fluorescence intensity into the two selected spectral bands and processes the signals (Figure 1). A laser Doppler velocimetry (LDV) optical device, providing two intersecting laser beams issuing

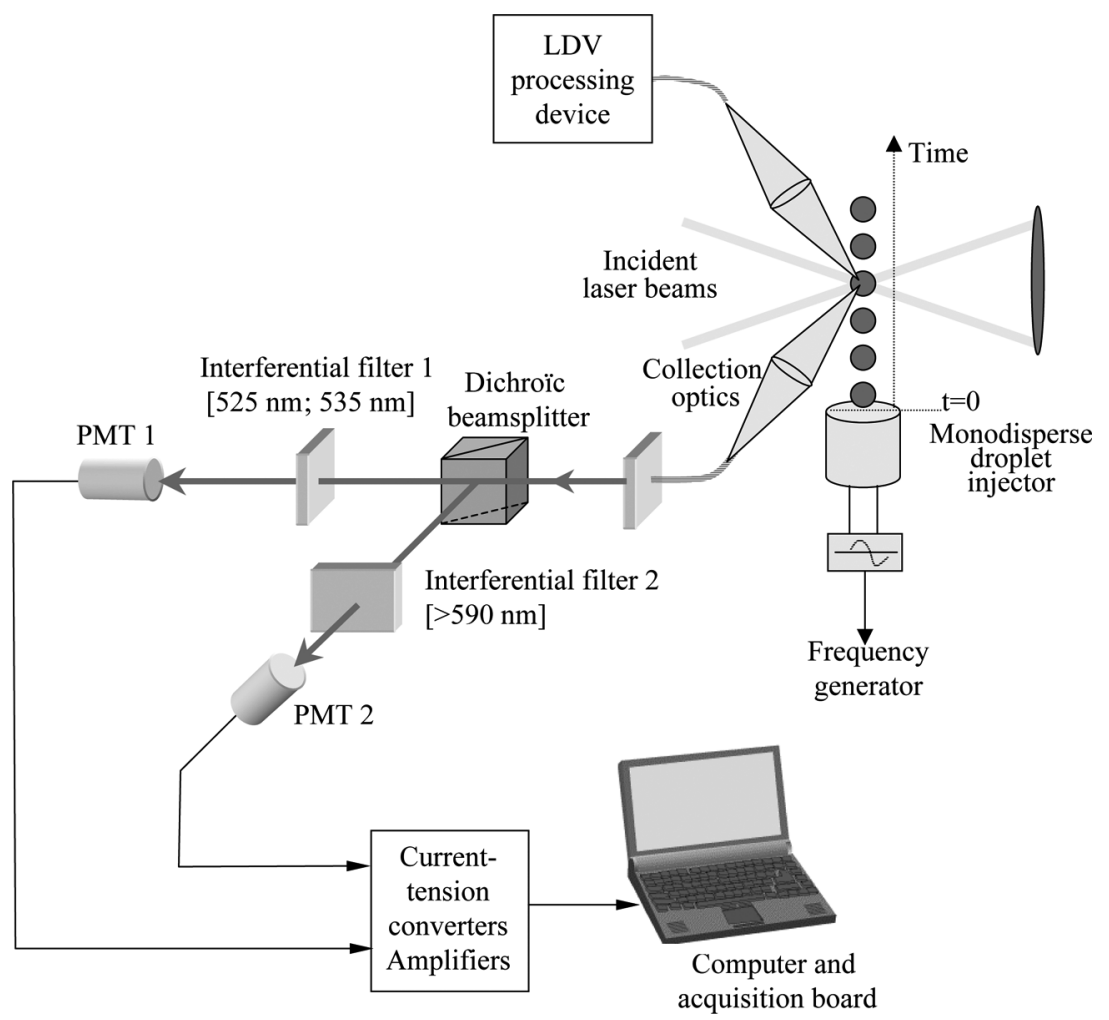

Figure 1. Block diagram of the experimental set-up: incident laser beams (LDV device) and LIF measurement set-up. 
from the same laser source has been used in order to create the probe volume (Figure 1). A mean droplet temperature can be obtained by using a probe volume diameter comparable to the droplet diameter itself and by averaging the fluorescence signal on the overall transit time of the droplet in the probe volume (Lavielle et al., 2001a, 2002). The main advantage of using a LDV optical setup is that the droplet velocity can be easily measured, which enables to determine the time elapsed from the injection point.

The fluorescence signal is collected at right angle by means of an achromatic doublet connected to an optical fiber. The fluorescence signal is transmitted by the optical fiber to a set of beamsplitters and optical filters (Figure 1), which enables the division of the fluorescence signal into the two specified spectral bands (band 1: [525 nm; $535 \mathrm{~nm}$ ]; band 2: $[575 \mathrm{~nm} ; 700 \mathrm{~nm}]$ ), as indicated earlier. (see also Lavieille et al. 2001a). The laser light scattering on the droplets is eliminated by the use of a notch filter (Optical density 6 at $\lambda=514.5 \mathrm{~nm}$ ). The fluorescence signal is detected on the two spectral bands by means of two photomultiplier tubes equipped with two rapid pre-amplifiers and finally the signal is sent to a rapid acquisition board.

The measuring volume dimensions for the fluorescence signal are given by the product of the excitation field by the detection field of view. Hence, the probe volume can be defined as the intersection of the three following volume:

- Laser excitation volume, which is usually defined as an ellipsoid, corresponding to the area of intersection of the two incident laser beams. With the used optics, the probe volume dimensions are $4000 \mu \mathrm{m}$ in the laser beam axis and $243 \mu \mathrm{m}$ for the transverse dimensions.

- Fluorescence collection volume, which can be defined by the image of the optical fiber core formed by the achromatic doublet on the laser excitation volume. According to the selected focal length, this image is a $200-\mu \mathrm{m}$ circle, which tends to reduce due to the lensing effect of the droplet.

- Droplet volume.

The probe volume is larger than the droplet sizes considered in the present work.

The droplet detection is based on an initial determination of a threshold on each spectral band of detection (Lavieille et al., 2001a). 
The threshold is fixed at a high value and is reevaluated at each measurement locations according to the turbulence level, in order to take up only the droplet well centered in the probe volume and hence to get a reasonable evaluation of the droplet mean temperature. The maximum toler-

ated eccentricity is estimated at $20 \mu \mathrm{m}$, which should not affect significantly the calculation of the average value on the whole droplet transit in the probe volume. Nevertheless, multiple realizations of a temperature measurement at a designated measurement location lead to a $\pm 1^{\circ} \mathrm{C}$ dispersion of the average temperature.

\section{PRINCIPLES OF THE COMBUSTION EXPERIMENT OF MONODISPERSE DROPLETS}

A linear monodisperse droplet stream is generated by Rayleigh disintegration of a liquid jet, with the use of a mechanical vibration obtained by a piezoceramic, excited by a square wave. The applied voltage on the piezoceramic depends on the desired position of the breakup zone and on the fuel physical properties, related to the injection temperature. For a given frequency, the liquid jet breaks up into equally spaced and monosized droplets, at the frequency of the forced mechanical vibration (Figure 2). The liquid fuel, is pressurized with compressed air between 0.2 and 0.8 bars and is forced through a calibrated orifice ranging from $\Phi_{0}=50 \mu \mathrm{m}$ to $\Phi_{0}=100 \mu \mathrm{m}$ in diameter. According to the Rayleigh theory, the resulting droplet diameter is about $1.9 \Phi_{0}$. For the present experiment, a $50 \mu \mathrm{m}$ and a $100 \mu \mathrm{m}$ hole diameter have been used. The droplet injection velocity can range from $2 \mathrm{~m} / \mathrm{s}$ to about $10 \mathrm{~m} / \mathrm{s}$. The observation of the fluorescence signal allows one to be sure that no satellite droplets form. The droplet initial diameter is controlled by measuring the fuel flowrate and the piezoceramic frequency. According to the accuracy of the measurements of the flowrate and piezoceramic frequency, the resulting accuracy on the initial diameter control is $\pm 0.5 \%$.

The fuel can be pre-heated in the injector body by means of an external heated water circulation. The temperature of the fuel is measured exactly at the injection point with the use of a K-type thermocouple. An electrically heated coil allows initiating the combustion just after the break-up zone of the liquid jet and a laminar flame appears (Figure 2). 


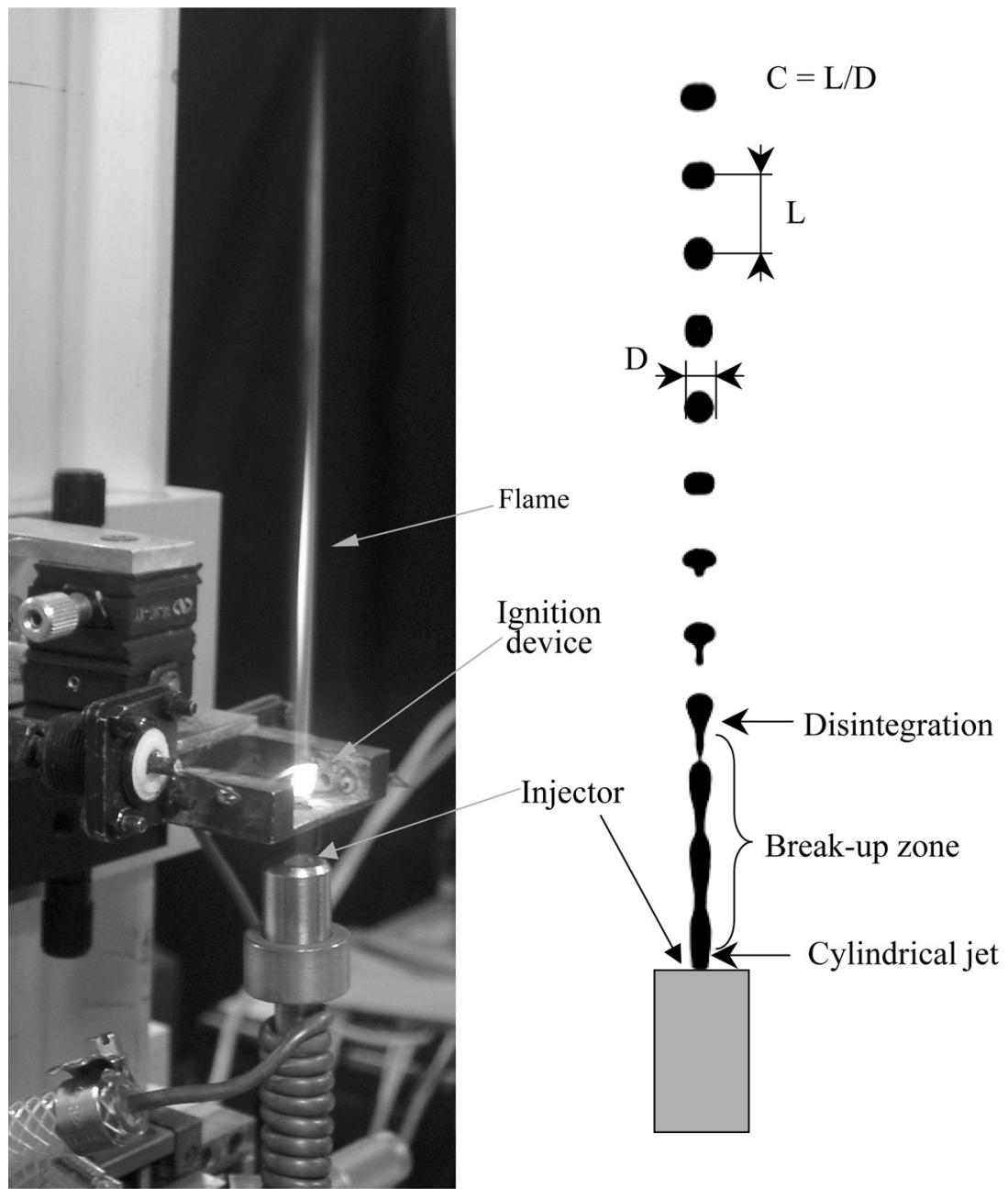

Figure 2. Picture of the monodisperse droplet stream in combustion.

\section{EXPERIMENTAL RESULTS}

The linear droplet stream has been explored point by point, from the ignition point. The exploration is stopped when the turbulent motions of the droplets become too important in the probe volume. The influence of two parameters has been considered: the distance parameter $\mathrm{C}$ and the droplet diameter D. Due to the operating constraints of the piezo-ceramic monodisperse injector, the fine tuning of these parameters 
requires also to vary the injection velocity. The exploration of a wide range of distance parameters is realized in two ways : modification of the piezofrequency for the moderate change, inducing a subsequent modification of the diameter, or electrostatic deviation (Lavieille et al., 2002) for the larger distance parameter, without initial droplet size changes.

A typical heating profile of combusting droplets in linear stream is shown in Figure 3. The injection velocity is $4.5 \mathrm{~m} / \mathrm{s}$, the droplet diameter $\mathrm{D}$ is $134 \mu \mathrm{m}$ and the distance parameter $\mathrm{C}=3.3$. Two phases may be clearly distinguished: the first phase corresponds to the transient heating of the droplet, where the temperature field is not homogeneous inside the droplet. The second one corresponds to an equilibrium phase : the inside droplet temperature field is now homogeneous and the droplet evaporates at a constant temperature, around $60^{\circ} \mathrm{C}$, well bellow the fuel boiling point at the atmospheric pressure $\left(78^{\circ} \mathrm{C}\right.$ for ethanol), corresponding to the wet bulb temperature. This temperature is the result of the equilibrium of the convective heat flux coming from hot gases surrounding the droplets and the heat drained for vaporization.

These kind of results are in agreement with previous numerical works; for example, Gutheil (1995), using an infinite conduction model,

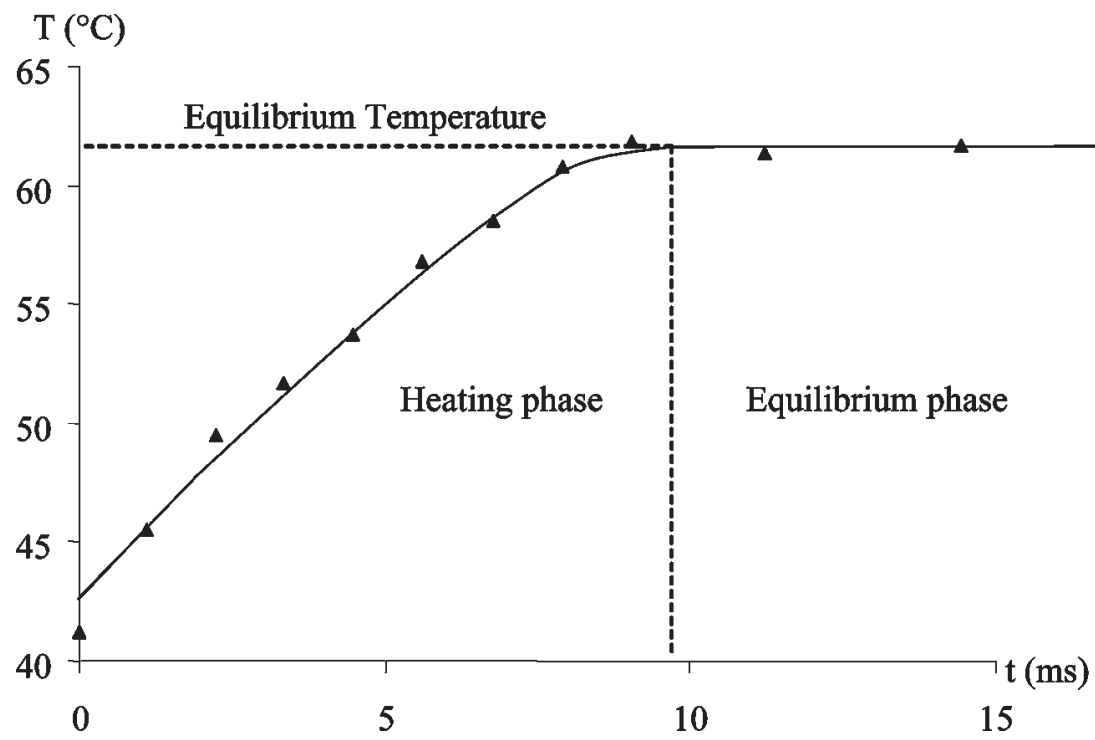

Figure 3. Typical heating profile of a combusting droplet $\left(V_{i}=4.5 \mathrm{~m} / \mathrm{s}, \mathrm{C}=3.3\right.$, $\mathrm{D}=134.4 \mu \mathrm{m})$. 
has calculated the temperature evolution of combusting droplets for several fuel species, like methanol, ethanol, n-heptane, n-octane, injected in a hot gaseous medium. The predicted equilibrium temperature, in the case of ethanol droplets, was also in the vicinity of $60^{\circ} \mathrm{C}$, very similar to the temperature found in the present work.

A full experimental database has been collected, with several aerothermal initial conditions, mainly defined by the droplet diameter, the distance parameter and the droplet injection velocity. These conditions are summarized in Table 1. For all the tested experimental conditions, the equilibrium temperature of the droplets ranges from about $58^{\circ} \mathrm{C}$ to $61^{\circ} \mathrm{C}$. The uncertainty of the LIF technique in combustion is $\pm 1^{\circ} \mathrm{C}$ : consequently, all the measured equilibrium temperature are very close, in the vicinity of $60^{\circ} \mathrm{C}$ and seem not to depend strongly on the aerothermal conditions.

\section{DROPLET HEATING MODELING}

The droplet heating is the result of the heat release from the combusting fuel vapor, creating a hot gas environment surrounding the droplets. Heat transfer occurs at the droplet surface due to forced convection. Two combined mechanisms are generally involved to explain the heat transfer inside the moving liquid droplets: the pure heat conduction and the advection by the internal motions caused by the friction phenomena between the liquid droplet surface and the external air flow. One may reasonably assume that the surface temperature reaches the equilibrium temperature a very short time after the ignition due to the high temperature of the gaseous phase, and the transient temperature field calculation turns out to the resolution of the heat diffusion equation, thus with a uniform and constant surface temperature.

Thus, the general assumptions of the heating model are:

- The temperature distribution along the droplet surface is uniformly distributed and equal to the observed equilibrium temperature,

- The effects of the moving droplet boundary due to vaporization are taken into account since the droplet geometry (radius) is modified at each time step of the resolution. As a consequence, the within droplet temperature field can be calculated by a succession of states where velocity and temperature fields are rescaled at each time step of the resolution, to match with updated droplet diameter. In so far, the droplet mass and heat capacity is reduced at each time step. 


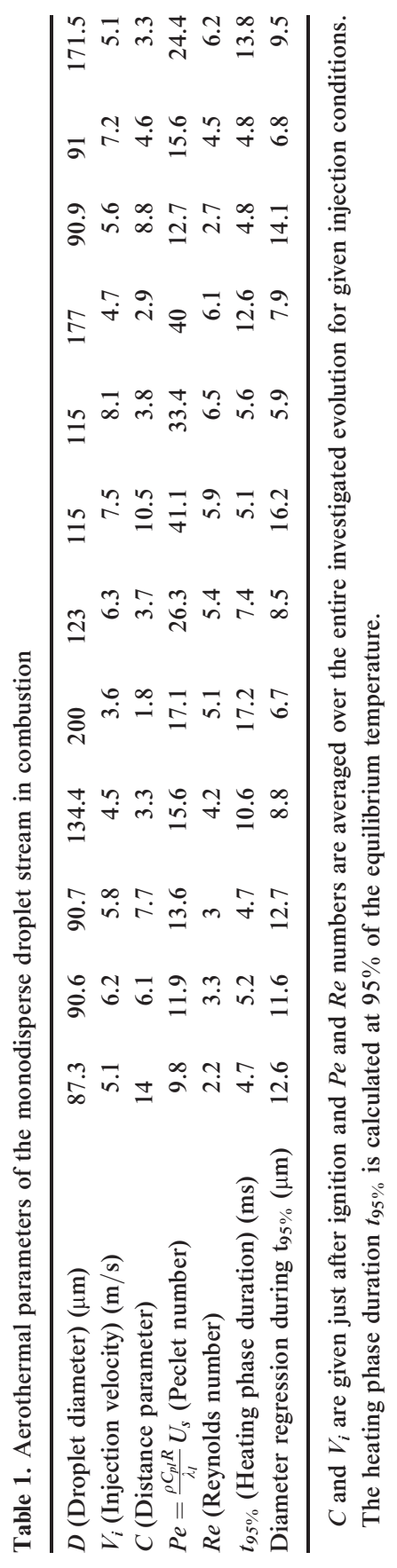


- The instantaneous velocity field inside the streaming droplet is approximated by the Hill spherical vortex solution (Abramzon and Sirignano, 1989), which is the rigorous solution for a non evaporating droplet.

In the same way, the temperature field can be calculated by resolution of the heat transfer equation, for a non-vaporizing droplet. Neglecting the viscous dissipation, and omitting the pseudo-convection term due to the moving boundary, under the quasi-steady hypothesis, this equation is:

$$
\frac{\partial T^{*}}{\partial t^{*}}+\overrightarrow{V^{*}} \cdot \overrightarrow{\operatorname{grad}^{*}} T^{*}=\frac{1}{P e} \Delta^{*} T^{*}
$$

In the dimensionless Eq. (3), the spatial coordinates are reduced by the droplet radius, considered as constant for a given time step.

The temperature $T$ is reduced by the surface temperature $T_{S}$ and the velocity components by the maximum velocity $U_{S}$ at the droplet surface.

The dimensionless time is defined as follows:

$$
t^{*}=\frac{U_{S}}{R} t
$$

Finally, the Peclet number $P e$ can be written:

$$
P e=\frac{\rho C_{p l} R}{\lambda_{l}} U_{S}
$$

The Hill vortex velocity field can be defined by its stream function $\psi$ :

$$
\psi\left(r^{*}, \theta, \varphi\right)=-\frac{\bar{A} R^{4}}{2} r^{* 2}\left(1-r^{* 2}\right) \sin ^{2} \theta
$$

where $\left(\boldsymbol{r}^{*}, \theta, \varphi\right)$ are the spherical coordinates of a point of the droplet, and $r^{*}=r / R$ is the dimensionless radius. $\bar{A}$, the vortex intensity, can be fixed by the maximum value of the velocity $U_{S}$ at the droplet surface:

$$
\bar{A}=\frac{U_{S}}{R^{2}}
$$

The resulting velocity vector components are in the $\left(r^{*}, \theta, \varphi\right)$ system (Figure 4):

$$
\overrightarrow{V^{*}}=\mid \begin{gathered}
\left(1-r^{* 2}\right) \cos \theta \\
-\left(1-2 r^{* 2}\right) \sin \theta \\
0
\end{gathered}
$$




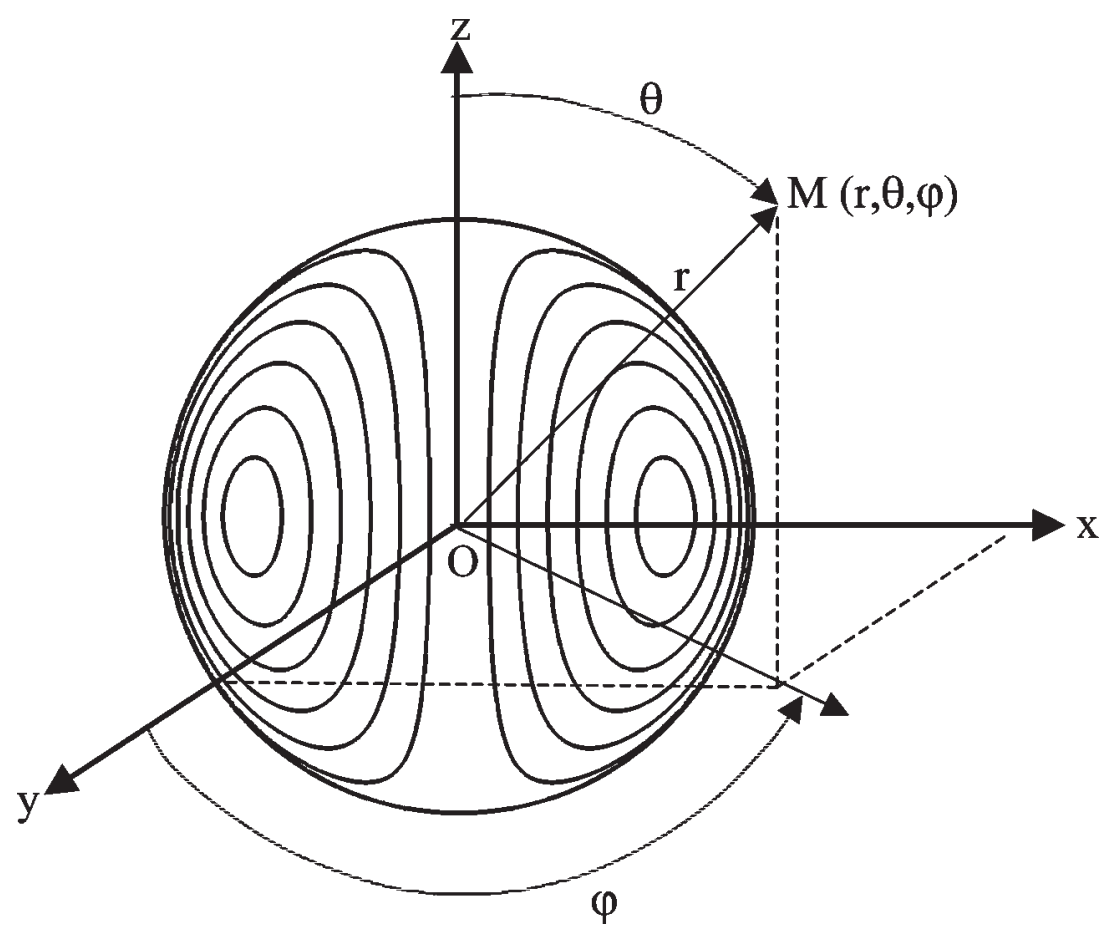

Figure 4. Streamlines of the inside droplet circulation according to the Hill vortex solution.

Taking into consideration the cylindrical symmetry around the $\mathrm{z}$ axis, the temperature in independent on the coordinate $\varphi$. The dimensionless temperature can be split on the Legendre polynomials base, according to:

$$
T^{*}\left(r^{*}, \theta, t\right)=\sum_{n=0}^{+\infty} f_{n}\left(r^{*}, t\right) P_{n}(\cos \theta)
$$

where $P_{n}(\cos \theta)$ are the Legendre polynomials and $f_{n}\left(r^{*}, t\right)$ are the associated harmonic functions, which should be determined according to Eq. (3), with $x=\cos \theta$ :

$$
\begin{aligned}
& \sum_{n=0}^{+\infty}\left[\left(\frac{\partial f_{n}}{\partial t^{*}}+\left(1-r^{* 2}\right) x \frac{\partial f_{n}}{\partial r^{*}}\right) P_{n}(x)+\frac{\left(1-2 r^{* 2}\right)}{r^{*}}\left(1-x^{2}\right) f_{n} P_{n}^{\prime}(x)\right] \\
& \quad=\frac{1}{P e} \sum_{n=0}^{+\infty}\left[\left(\frac{\partial^{2} f_{n}}{\partial r^{* 2}}+\frac{2}{r^{*}} \frac{\partial f_{n}}{\partial r^{*}}\right) P_{n}(x)+\frac{1}{r^{* 2}} f_{n}\left(\left(1-x^{2}\right) P_{n}^{\prime \prime}(x)-2 x P_{n}^{\prime}(x)\right)\right]
\end{aligned}
$$


The Legendre polynomials are solutions of the following differential equation:

$$
\left(1-x^{2}\right) P_{n}^{\prime \prime}(x)-2 x P_{n}^{\prime}(x)+n(n+1) P_{n}(x)=0
$$

In the light of Eq. (11), Eq. (10) may be rewritten:

$$
\begin{aligned}
& \sum_{n=0}^{+\infty}\left[\left(\frac{\partial f_{n}}{\partial t^{*}}+\left(1-r^{* 2}\right) x \frac{\partial f_{n}}{\partial r^{*}}\right) P_{n}(x)+\frac{\left(1-2 r^{* 2}\right)}{r^{*}}\left(1-x^{2}\right) f_{n} P_{n}^{\prime}(x)\right] \\
& \quad=\frac{1}{P e} \sum_{n=0}^{+\infty}\left[\left(\frac{\partial^{2} f_{n}}{\partial r^{* 2}}+\frac{2}{r^{*}} \frac{\partial f_{n}}{\partial r^{*}}-\frac{n(n+1)}{r^{* 2}} f_{n}\right) P_{n}(x)\right]
\end{aligned}
$$

Multiplying Eq. (12) by $P_{m}(x)$, integrating between -1 and +1 and taking into account the Legendre polynomials orthogonality property, allows one to determine the equation verified by the rank $\mathrm{m}$ harmonic function $f_{m}$ :

$$
\begin{aligned}
\frac{\partial f_{m}}{\partial t^{*}} & +\frac{1}{C_{m}} \sum_{n=0}^{+\infty}\left[A_{n m}\left(1-r^{* 2}\right) \frac{\partial f_{n}}{\partial r^{*}}+B_{n m} \frac{1-2 r^{* 2}}{r^{*}} f_{n}\right] \\
& =\frac{1}{P e}\left(\frac{\partial^{2} f_{m}}{\partial r^{* 2}}+\frac{2}{r^{*}} \frac{\partial f_{m}}{\partial r}-\frac{m(m+1)}{r^{* 2}} f_{m}\right)
\end{aligned}
$$

where:

$$
\begin{gathered}
A_{n m}=\int_{-1}^{+1} x P_{n}(x) P_{m}(x) d x \\
B_{n m}=\int_{-1}^{+1}\left(1-x^{2}\right) P_{n}^{\prime}(x) P_{m}(x) d x
\end{gathered}
$$

and

$$
C_{m}=\frac{1}{m+(1 / 2)}
$$

$A_{n m}$ and $B_{n m}$ are calculated.

\section{PRINCIPLES OF THE NUMERICAL SIMULATION AND OF THE COMPARISON TO EXPERIMENTAL DATA}

The calculation of the droplet temperature field is performed under a quasi-steady assumption: at each time step $(0.1 \mathrm{~ms})$, the droplet 
diameter, velocity, Peclet number are updated as well as the physical properties of the gas phase.

\section{Droplet Velocity and Diameter Evolution}

The physical properties of the gas phase are calculated at the reference state, updated at each time step, according to the " $1 / 3$ rule". The reference conditions are defined by:

$$
\begin{gathered}
T_{r e f}=T_{S}+\left(T_{a}-T_{S}\right) / \mathbf{3}, \text { for the temperature } \\
Y_{F r e f}=Y_{F S}+\left(Y_{F \infty}-Y_{F S}\right) / \mathbf{3}, \text { for the fuel mass fraction }
\end{gathered}
$$

The ambient temperature has been measured by the CARS technique on the droplet stream axis for different distance parameters (see Figure 5, from Virepinte et al., 2000).

The droplet velocity is calculated at each time step, according to dynamic equation, neglecting the pressure gradients and history forces for droplets streaming on a linear vertical trajectory in quiescent air:

$$
\frac{V(t+\delta t)-V(t)}{\delta t}=\frac{3 \rho_{g} C_{D}}{4 \rho_{1} D} V^{2}(t)+g
$$

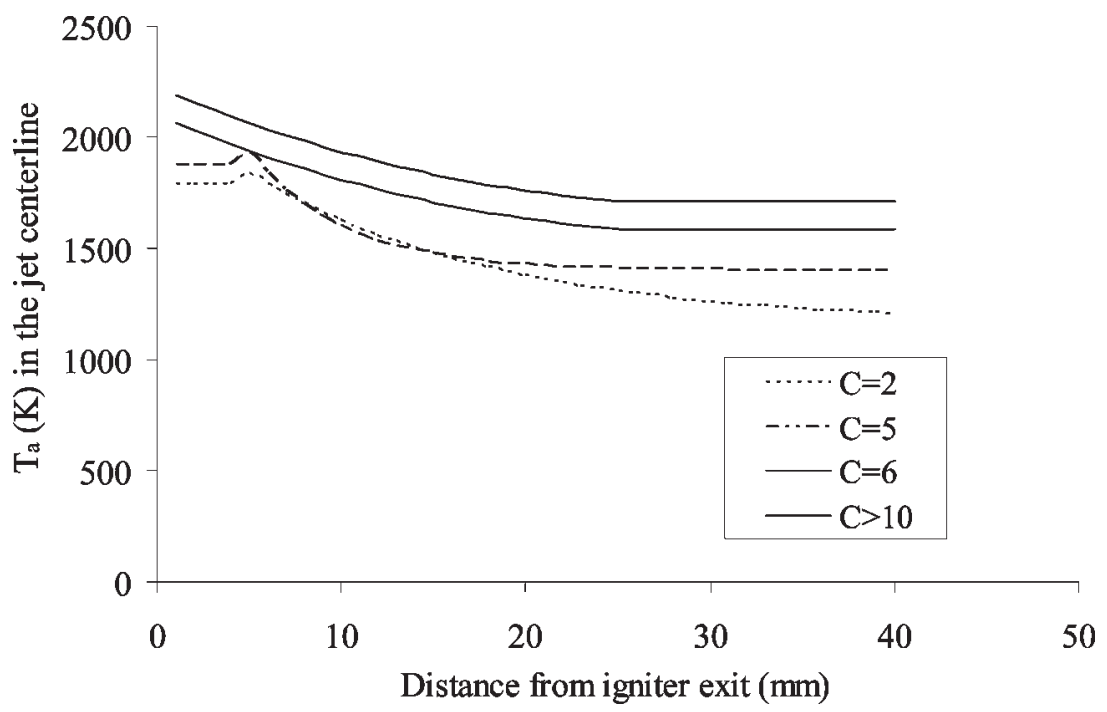

Figure 5. Gas phase temperature in the jet centerline measured by CARS thermometry (from Virepinte et al. (2000)). 
where $V(t)$ is the velocity at the instant $\mathrm{t}, V(t+\delta t)$ is the velocity at instant $t+\delta t$ and $C_{D}$ is the drag coefficient. For the drag coefficient $C_{D}$, the correlation of Virepinte et al. (2000), determined in similar conditions is used:

$$
C_{D}=3 \mathrm{Re}^{-0.92} C^{0.59}
$$

The diameter regression due to evaporation is calculated at each time step, according the correlation developed by Abramzon and Sirignano (1989) and Sirignano (1999), for the vaporization mass flux of an isolated moving droplet:

$$
\begin{aligned}
\dot{m}= & \pi \rho a D \log \left(1+B_{M}\right) \\
& \times\left[\frac{2 F\left(B_{M}\right)+(1+\mathrm{Re} S c)^{1 / 3} \max \left(1, \mathrm{Re}^{0.077}\right)-1}{F\left(B_{M}\right)}\right]
\end{aligned}
$$

$a$ being the diffusivity of the fuel vapor in air.

The droplet Reynolds number is calculated as suggested by Abramzon and Sirignano (1989) with the specific density taken at the flame temperature, since the droplets are moving in a column-shaped flame and neglecting the gas-phase velocity compared to the droplet velocity:

$$
\operatorname{Re}=\frac{\rho_{g}\left(T_{a}\right) V D}{\mu\left(T_{r e f}\right)}
$$

$B_{M}$ is the Spalding mass transfer number defined by:

$$
B_{M}=\frac{Y_{F S}-Y_{F \infty}+\nu t Y_{o x \infty}}{1-Y_{F S}}
$$

where $Y_{F S}$ and $Y_{F \infty}$ are the vapor fuel mass fraction at the droplet surface and far from the jet respectively $\left(Y_{F \infty}=0\right)$ and $Y_{o x \infty}$ is the mass fraction of the oxidizer far from the jet. $\nu$ is the stoichiometric coefficient.

According to Sirignano (1999), $F\left(B_{M}\right)$ is given by:

$$
F\left(B_{M}\right)=\left(1+B_{M}\right)^{0.7} \frac{\log \left(1+B_{M}\right)}{B_{M}}
$$

For interacting droplets in linear stream, Virepinte et al. (2000) recommended the use of a coefficient $\eta$ depending on the distance parameter $\mathrm{C}$ only, based on experimental investigations performed in similar conditions, in order to correct the vaporization mass flux:

$$
\dot{m}_{c o r}=\eta(C) \dot{m}
$$


where $\dot{m}_{c o r}$ is the evaporation mass flux corrected by the interaction phenomena, $\dot{m}$ being established for an isolated droplet. For all the tested conditions, the droplet diameter regression during the transient heating phase has been calculated; the heating phase duration as well as the diameter regression are reported in Table 1. The duration of the transient heating phase is calculated when the temperature reaches $95 \%$ of the equilibrium temperature. The diameter reduction between two time steps does not exceed $0.3 \mu \mathrm{m}$. It is clear that the vaporization rate is strongly influenced by the factor $\eta$, which can reach 0.23 for $C=2$ and 0.92 for $C=8$.

\section{Initial and Boundary Conditions}

In the light of the experimental data, the droplet surface temperature $T_{S}$ will be fixed at the equilibrium temperature $T_{e q}$ observed in the experimental investigations;

$$
\forall \theta,\left\{\begin{array}{l}
f_{0}(r=R, t)=T_{S}=T_{e q} \\
\forall n \geq 1, f_{n}(r=R, t)=0
\end{array}\right.
$$

The second boundary condition is defined to ensure the spatial continuity of the heat flux in the droplet center:

$$
\forall n \geq 0, \quad \frac{d f_{n}}{d r}(r=0, t)=0
$$

The initial condition is more subject to discussion, since it is related to the history of the droplet before its ignition. The simulations will be conducted from the ignition point to the equilibrium. Thus, the initial condition must be specified at the ignition point, where the spherical symmetry assumption will be done. The droplet surface temperature can be reasonably assumed to be at the equilibrium temperature, and a part of the droplet volume, called droplet core, is supposed to remain at the ambient temperature $T_{\infty}$. Thus, the initial condition is split into a constant temperature segment (fixed at $T_{\infty}$ ), representing the droplet core and a parabolic evolution starting from $T_{\infty}$ and reaching the surface temperature, fixed at the equilibrium temperature (Figure 6):

$$
\left\{\begin{array}{l}
T_{0}=T_{c}=T_{\infty} \quad(r \leq a) \\
T_{0}=A(r-a)^{2}+B(r-a)+T_{\infty} \quad(a<r \leq R)
\end{array}\right.
$$

where $T_{\infty}$ is the ambient temperature, a is the abscissa of the link-up point (with continuity of the derivative) between the constant 


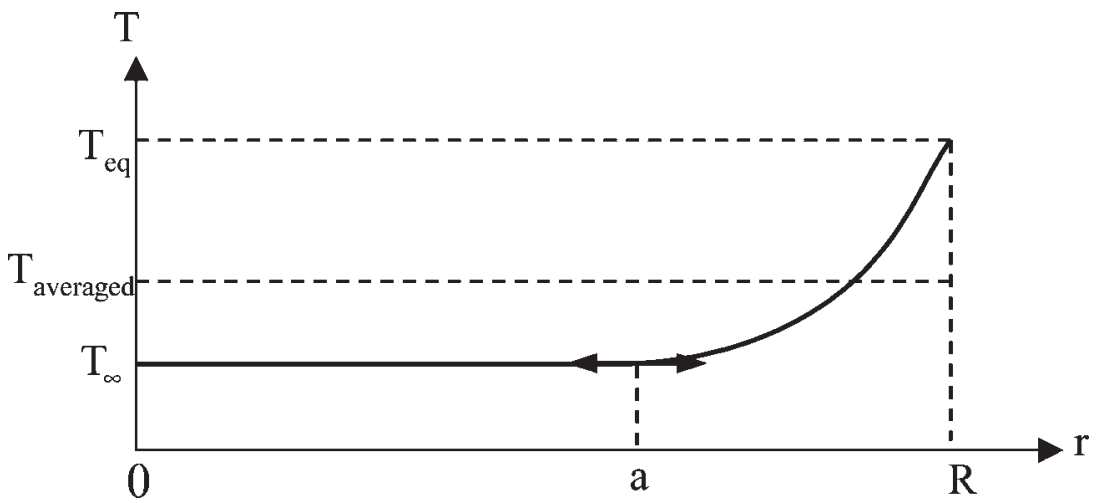

Figure 6. Typical initial condition for the temperature used in the simulation.

temperature segment and the parabolic evolution. The parameters a, A and $\mathrm{B}$ are fixed to match with the following conditions:

- Equilibrium temperature at the droplet surface $\left(r=R, T=T_{s}\right)$;

- Droplet core temperature at $T_{\infty}$;

- Space averaged temperature measured at the ignition point.

The sensitivity of the calculation to the initial condition will be the object of further discussions.

Hence, due to the spherical symmetry, the initial conditions for the harmonic functions are:

$$
\forall \theta,\left\{\begin{array}{l}
f_{0}(r, t=0)=T_{0}(r) \\
\forall \boldsymbol{n} \geq 1, f_{n}(r, t=0)=0
\end{array}\right.
$$

Figure 7 shows the spatial variation of the temperature field calculated with 20 terms in Eq. (9). The calculation was carried out for a $134-\mu \mathrm{m}$ diameter droplet at about the middle of the duration of the heating phase. After several tests, it has been shown that the difference between the mean temperature calculated with 10 and 20 terms was very small, less than $0.1^{\circ} \mathrm{C}$. Hence, the number of functions $f_{n}$ was limited to 20 .

\section{Theory and Experiments Comparison}

Discussion on the Maximum Surface Velocity Adjustment: With the preceding initial and boundary conditions fixed, the last adjustable 


\section{$\mathrm{T}\left({ }^{\circ} \mathrm{C}\right)$}

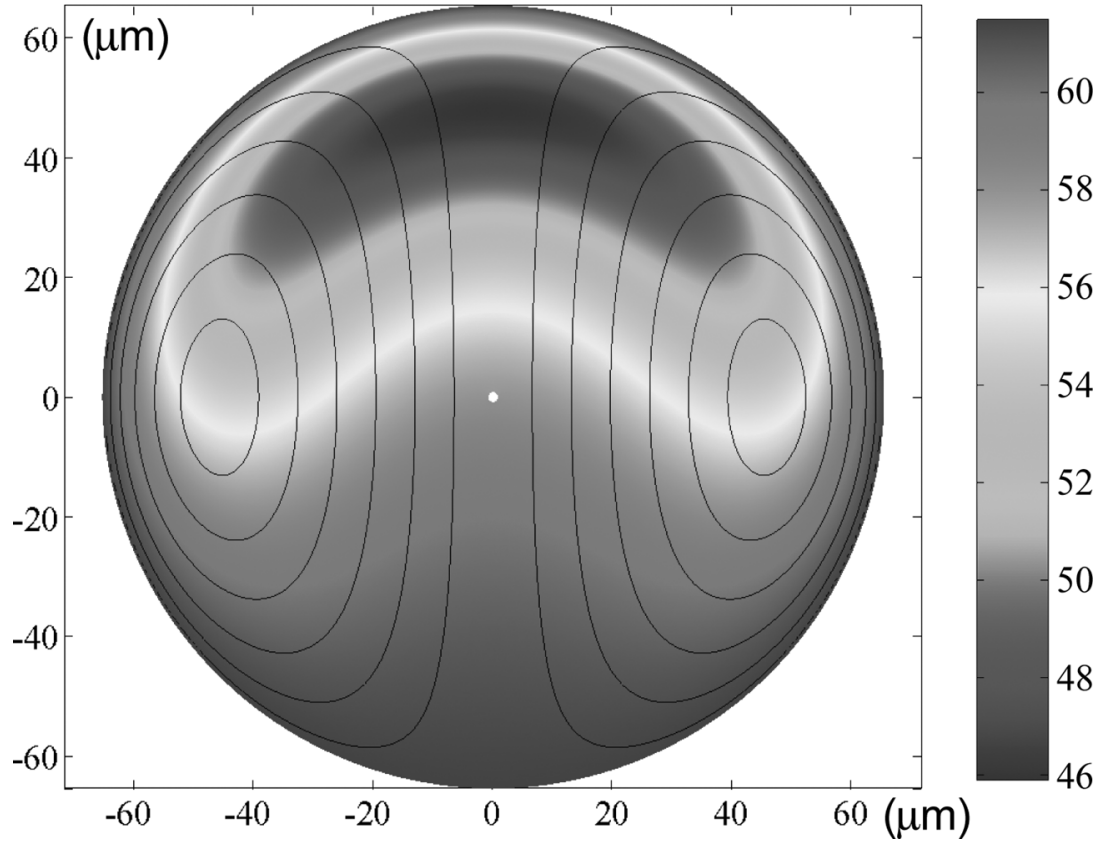

Figure 7. Calculated temperature field within a $134-\mu \mathrm{m}$ diameter droplet, calculated with 20 $f_{n}$ functions, at $\mathrm{t}=5.6 \mathrm{~ms}$. The streamlines are indicated.

variable is the maximum velocity at the droplet surface $U_{s}$, which is directly related the vortex intensity, according to Eq. (7). In light of the experimental results, $U_{s}$ is adjusted in order to obtain the best agreement between the numerical and experimental data. On the same example already used in earlier and with $U_{s}$ adjusted, a good agreement between the calculated heating profile of the droplets and the experimental data may be observed (Figure 8). It demonstrates that the Hill vortex model, associated with the selected boundary conditions is suitable for a fine description of the droplet heating phase. Furthermore, $U_{s}$ exhibits a moderate evolution as a function of time. In the next development, a unique value of $U_{s}$ averaged in time will be considered for given experimental conditions. The calculation with $\pm 25 \%$ of the selected value of $U_{s}$, also represented in Figure 8, shows that the choice of $U_{s}$ is not too critical, since the three curves appears very close. Furthermore, it justifies to take the mean value for $U_{s}$. 


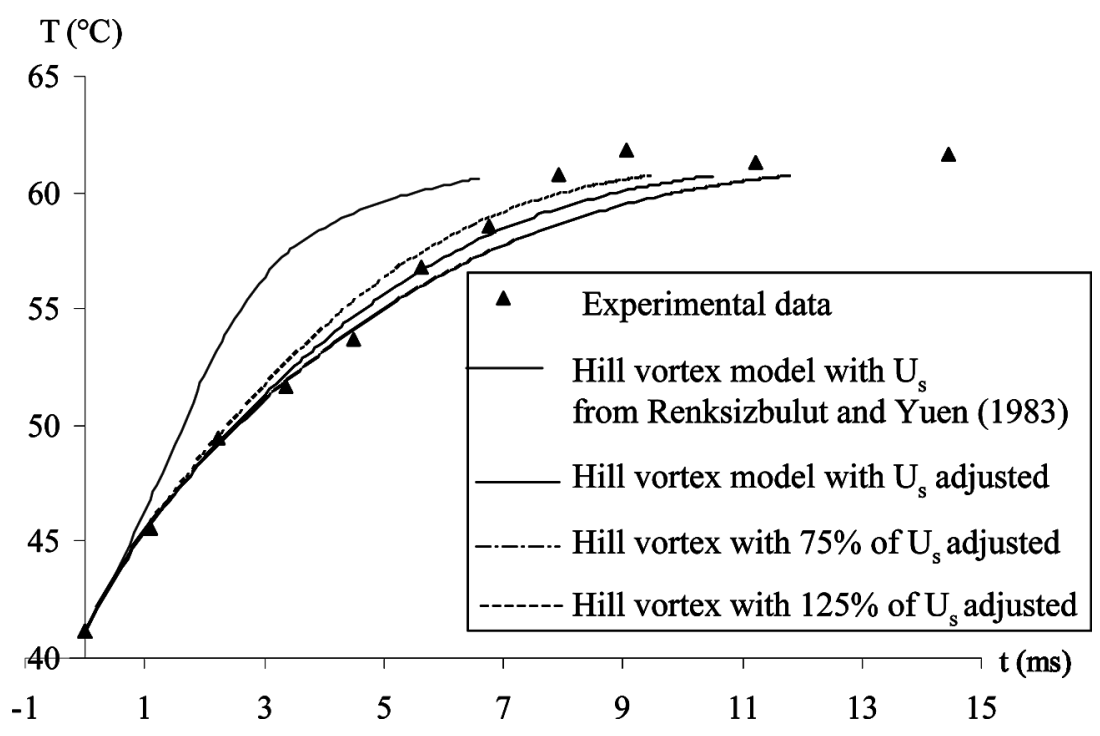

Figure 8. Sensitivity of the adjustment of $U_{s}$, comparison with the correlation of Rensizbulut and Yuen (1983) $(\mathrm{D}=134 \mu \mathrm{m} \mathrm{C}=3.3)$.

The effect of the initial condition should also be discussed on the same experimental example. The main problem is to fix the temperature of the droplet core, depending mainly on the droplet history between the liquid jet breakup and the ignition. The droplets are injected over the ambient temperature, but vaporization between injection and ignition occurs, which causes an initial cooling, the surface temperature remaining lower than the core in the first instants after the injection. A quasiinstantaneous reversal of the temperature gradients appears at the point of ignition and the surface attains the equilibrium temperature. In view of these preliminary remarks, the influence of the droplet core temperature, varying from about $15^{\circ} \mathrm{C}$ to $30^{\circ} \mathrm{C}$ have been tested, keeping in mind that the final calculations have been made with $T_{c}=T_{\infty}=20^{\circ} \mathrm{C}$. The calculation with the three initial conditions $\left(T_{c}=15^{\circ} \mathrm{C}, T_{c}=T_{\infty}=20^{\circ} \mathrm{C}\right.$, $\left.T_{c}=30^{\circ} \mathrm{C}\right)$ (Figure 9) are presented on the same example $(D=134 \mu \mathrm{m}$; $C=3.3$ ) in Figure 10. The curves corresponding to $T_{c}=15^{\circ} \mathrm{C}$ and $T_{c}=T_{\infty}=20^{\circ} \mathrm{C}$ are quite collapsing and very few differences are observed with $T_{c}=30^{\circ} \mathrm{C}$. Thus, it seems reasonable to select for the initial condition $T_{c}=T_{\infty}=20^{\circ} \mathrm{C}$. 


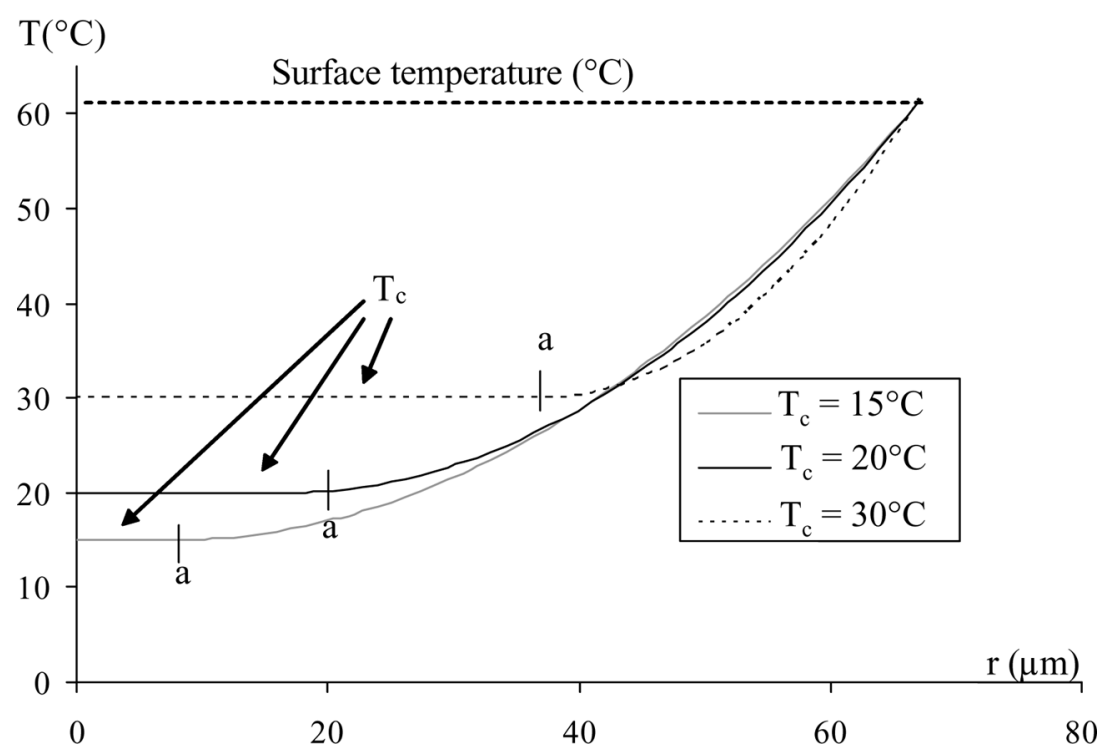

Figure 9. Sketch of the tested initial conditions.

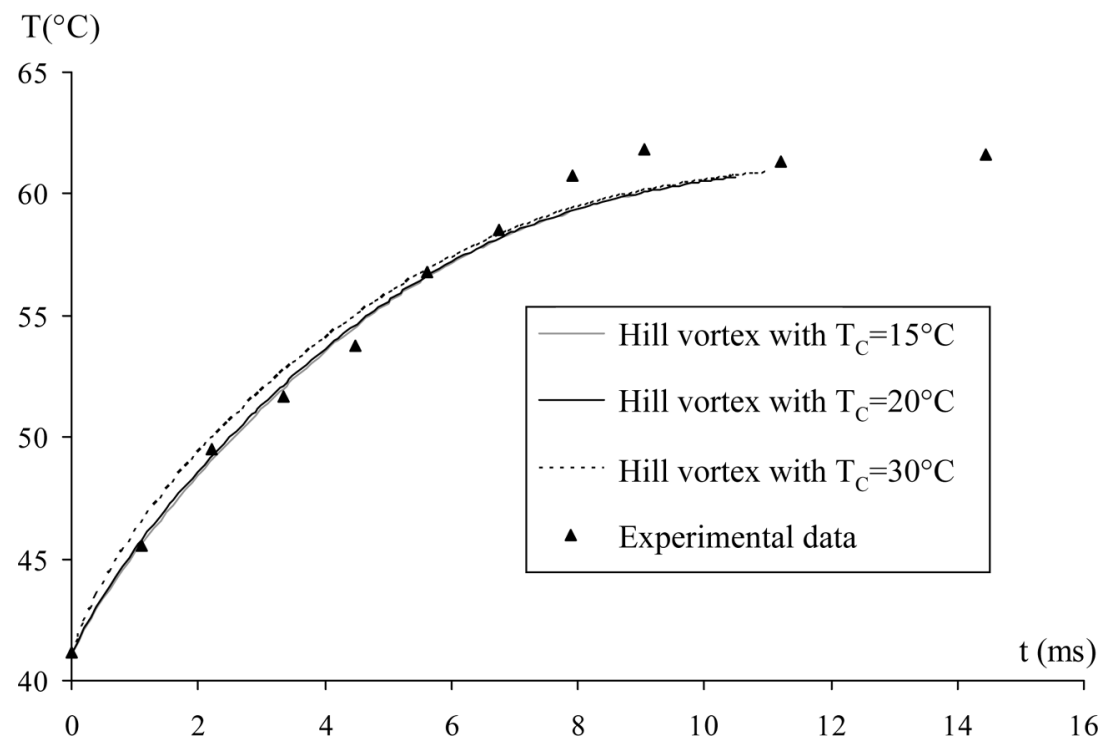

Figure 10. Influence of the initial condition: test of three core temperatures $(C=3.3$, $\mathrm{D}=134 \mu \mathrm{m})$. 
Experimental Data and Models Comparisons: The experimental data have been also compared to the effective conductivity model, first introduced by Jin and Borman (1985) and Talley and Yao (1986) in order to have a simplified calculation procedure for internally circulating droplets. The approach is similar to the conduction limit model, but an effective conductivity $k_{\text {eff }}$ is used instead of the regular thermal conductivity:

$$
k_{\text {eff }}=\chi k
$$

Abramzon and Sirignano (1989) found the following approximation for $\chi$ :

$$
\chi=1.86+0.86 \tanh \left[2.245 \log _{10}(P e / 30)\right]
$$

With the adjusted value of $U_{s}$, the corresponding Peclet number and subsequently the coefficient $\chi$ can be determined. The heating profiles calculated by the effective conductivity models is reported in Figure 11, for the same example. The curvature of the heating profile appears slightly different, but the time to reach the equilibrium is almost similar for both Hill vortex and effective conduction models.

The comparison with $U_{s}$ determined by the correlation of Abramzon and Sirignano (1989) and Sirignano (1999) is reported in Figure 11. From a theoretical point of view, this velocity is determined by the

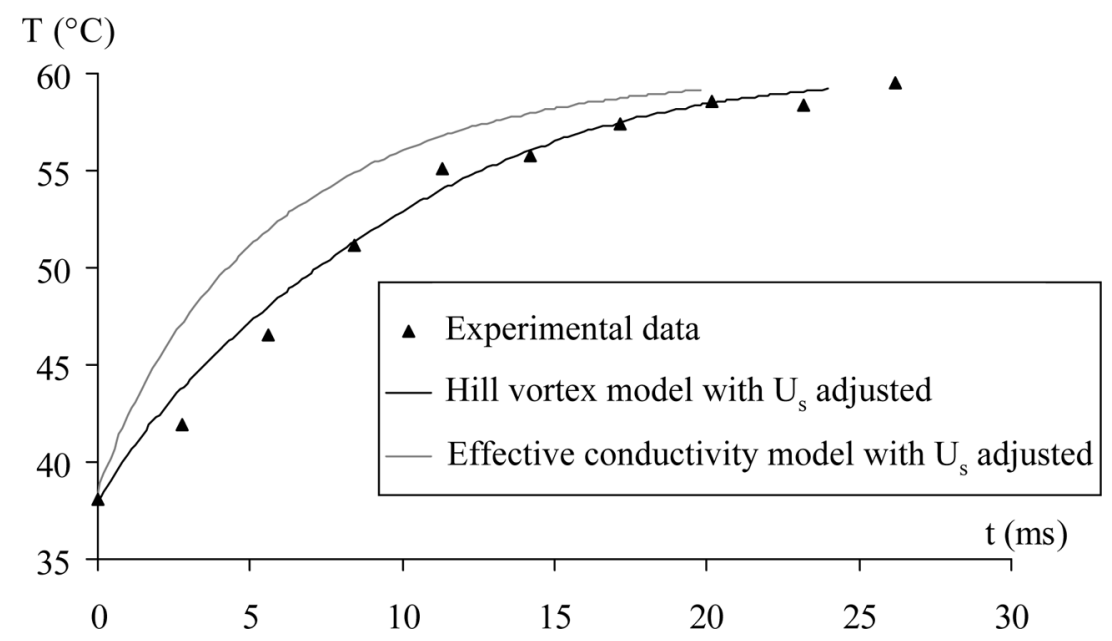

Figure 11. Triple comparasion between the effective conductivity model, the Hill vortex model and experimental data $(\mathrm{D}=134 \mu \mathrm{m}, \mathrm{C}=3.3)$. 
friction component of the droplet drag force, and according to Sirignano (1999), in the case of a moving isolated droplet, can be expressed by:

$$
U_{S}=\frac{1}{6 \pi}\left(V_{\infty}-V\right)\left(\frac{\mu_{g}}{\mu_{l}}\right) \operatorname{Re} C_{F}
$$

where $V_{\infty}$ is neglected in the present case. The Reynolds number is calculated according to Eq. (22).

Taking into account the drag reduction due to evaporation, the friction coefficient $C_{F}$ is written as suggested by Renksizbulut and Yuen (1983):

$$
C_{F}=\frac{12.69}{\operatorname{Re}^{2 / 3}\left(1+B_{M}\right)}
$$

However, the comparison of the experimental data fails clearly (Figure 8). One potential explanation is that this correlation was clearly established for an isolated droplet with radial symmetry properties and seems to be not applicable directly in the case of interacting droplets in linear stream, associated with a column shaped flame.

With the whole set of the collected experimental data (various distance parameters and Reynolds numbers), a correlation for the maximum surface velocity $U_{s}$ can be searched as an expression similar to Eq. (33):

$$
C_{F}=\frac{K}{\operatorname{Re}^{2 / 3}\left(1+B_{M}\right)}
$$

where $\mathrm{K}$ is a constant that should be adjusted in the light of the experimental data.

According to Eqs. (32) and (34), the resulting expression for droplets moving in a quiescent atmosphere $\left(V_{\infty}=0\right)$ of the maximum surface velocity $U_{s}$ is given by:

$$
\left|\frac{U_{s}}{V}\right|=\frac{1}{6 \pi}\left(\frac{\mu_{g}}{\mu_{l}}\right) K \frac{\operatorname{Re}^{1 / 3}}{\left(1+B_{M}\right)}
$$

The plot of $\mu_{l} / \mu_{g}\left(U_{s} / V\right)\left(1+B_{M}\right)$ as a function of $\mathrm{Re}^{1 / 3}$ enables the determination of the constant $K$ by the least squares method (Figure 12). The value of $K$ is approximately 4.9, which is about 2.5 times smaller than the value found by Renksizbulut and Yuen (1983) (Eq. (33)).

Furthermore, the careful observation of Figure 12 shows that there is no significant observable effect of the distance parameter, ranging in the present experimental database from 1.62 to 12.7 . The droplets to droplets interactions are probably present, but their effect on the inside 


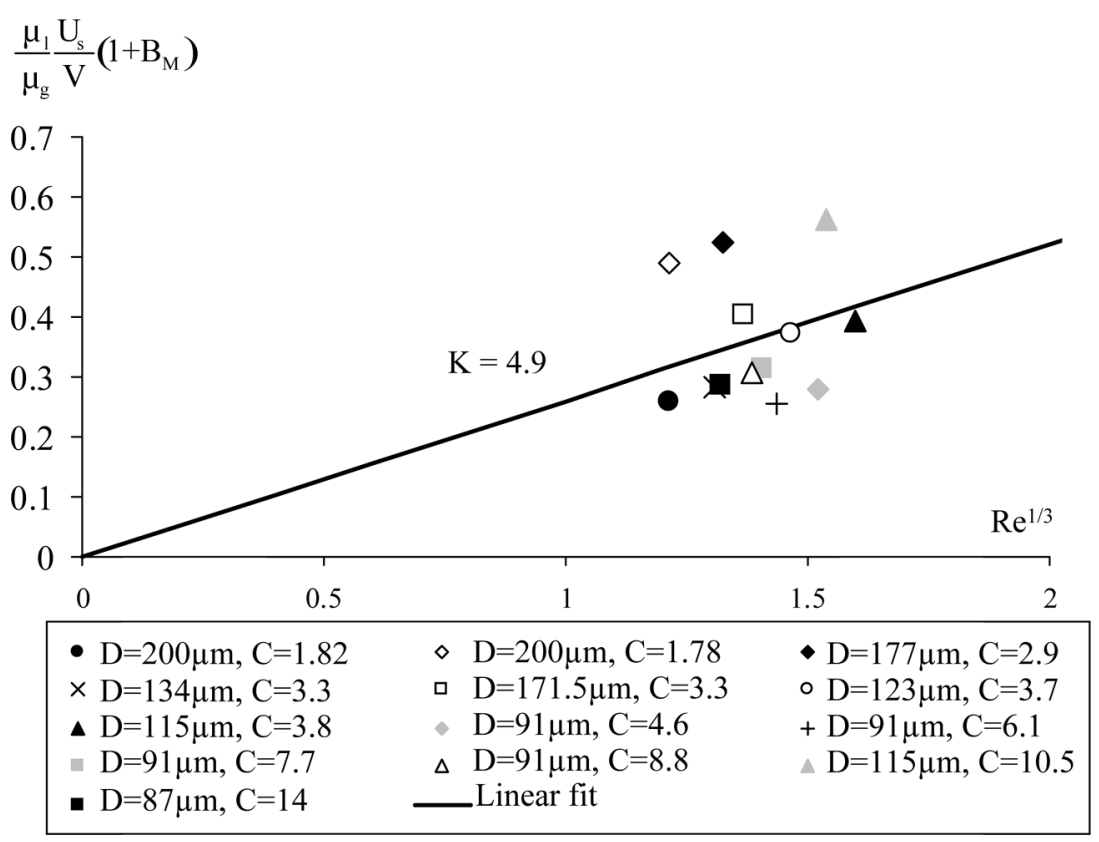

Figure 12. Adjustment of the surface velocity as a function of the Re number, influence of the distance parameter.

droplet heat transfer seems to be out of the sensitivity of the implemented experimental techniques.

The good agreement of the model with the complete experimental database set is obvious when both calculations with $U_{s}$ fitted and the experimental data are plotted together (Figure 13), in non dimensional coordinates. The time is reduced according to Eq. (4), and due to the wide range of the injection temperatures, the droplet temperatures are reduced by the following way:

$$
T^{*}=1-\left(\frac{T_{e q}-T}{\Delta T}\right)
$$

where $\Delta T$ is the less common temperature increase overall the tested experimental conditions. The Peclet number based on $U_{s}$ calculated according to Eq. (35) appears sufficient to describe the heating profile of combusting droplets in linear stream. It may also be added that the indicated Peclet number is time averaged over the entire investigated evolution of the droplet, for given injection conditions. 


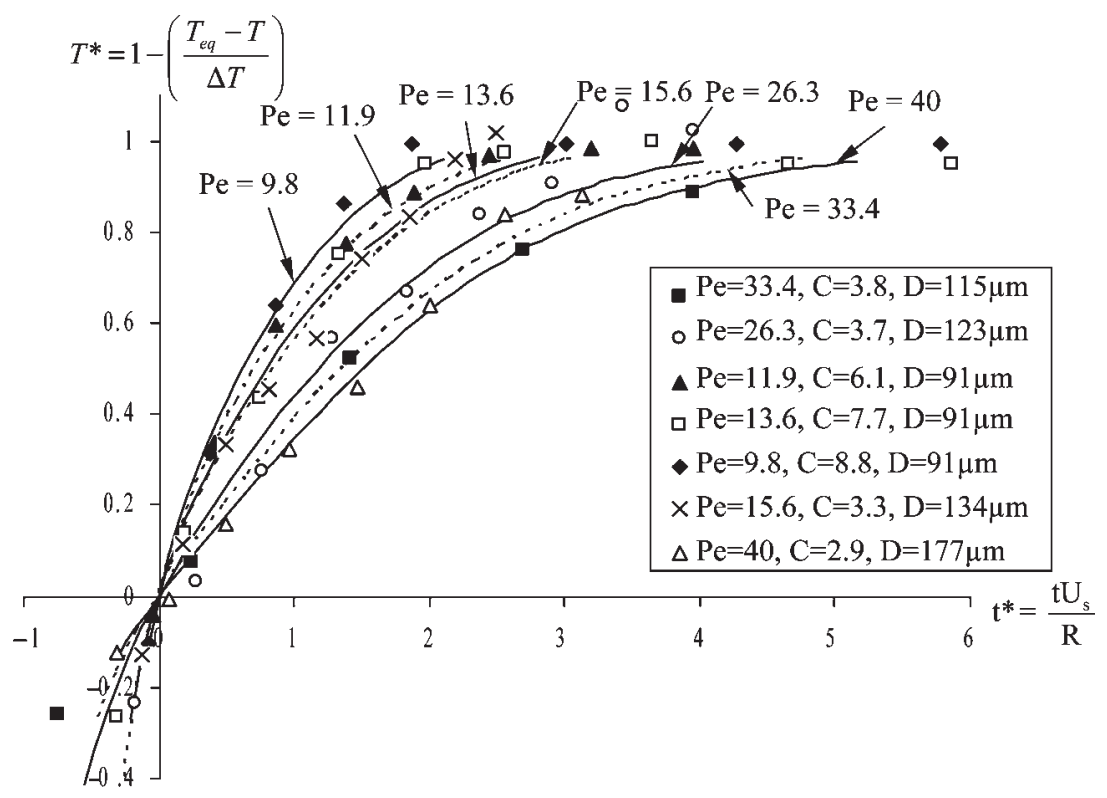

Figure 13. Experimental and calculated non dimensional droplet heating profiles for different Peclet numbers.

\section{CONCLUDING REMARKS}

The two colors laser-induced fluorescence technique has been applied to the characterization of the heating phase of combusting monodisperse droplets streaming linearly. A complete database, including a wide range of aerothermal injection parameters such as injection velocity, droplet diameter and inter-droplet distance (or non-dimensional distance parameter) has been provided. All the measurements have shown that the droplet thermal evolution can be split into two phases: a transient heating phase, followed by a constant temperature phase at a temperature under the boiling point of the fuel (equilibrium temperature), in the vicinity of $60^{\circ} \mathrm{C}$ in the case of ethanol. A calculation based on the heat diffusion equation resolution, with a constant temperature fixed at the equilibrium temperature has been implemented. Both heat conduction and heat advection by the droplet internal motions have been taken into account, the inside droplet velocity filed being modeled by the Hill vortex solution. The velocity reduction due to drag forces and the droplet diameter reduction due to vaporization are also included in the model. 
The resulting experimental heating profiles enable the determination of the Hill vortex intensity related to the maximum velocity at the droplet surface and to propose a modified correlation based on the previous theoretical work of Abramzon and Sirignano (1989) and Sirignano (1999), developed for isolated droplets. It is clearly highlighted that the Peclet number based on the maximum droplet surface velocity, calculated by the new correlation is sufficient to describe the droplets heating profiles. The distance parameter, in the investigated range $(1.62 \leq C \leq 12.7)$ seems not to have a significant influence, taking into consideration the actual accuracy and resolution of the measurement techniques. This influence exists probably for the smallest distance parameters and for larger diameters reduction during the heating phase, and this influence should be studied in further development of this work. From a more general point of view, the model should remain applicable in a dilute spray injected in a turbulent flow, if the turbulent scale is significantly smaller or bigger than the droplet size.

\section{NOMENCLATURE}

$\begin{array}{ll}a & \text { Fuel vapor diffusivity in air } \\ B_{M} & \text { Mass transfer Spalding number } \\ C & \begin{array}{l}\text { Molecular concentration of the fluorescent tracer or non } \\ \text { dimensional distance parameter, }\end{array} \\ C_{D} & \text { Drag coefficient } \\ C_{p} & \text { Specific heat } \\ D & \text { Droplet diameter } \\ f_{n} & \mathrm{n}^{\text {th }} \text { harmonic function in the Legendre polynomials basis } \\ g & \text { Gravity acceleration intensity } \\ I_{0} & \text { Incident laser intensity } \\ I_{f} & \text { Fluorescence intensity } \\ K_{\text {opt }} & \text { Optical constant } \\ K_{\text {spec }} & \text { Spectroscopic constant } \\ (\boldsymbol{r}, \theta, \varphi) & \text { Spherical coordinates system } \\ \dot{m} & \text { Vaporization mass flux } \\ P e & \text { Peclet number } \\ P_{n} & \text { n }{ }^{\text {th }} \text { Legendre polynomial } \\ R & \text { Droplet radius } \\ \operatorname{Re} & \text { Reynolds number }\end{array}$




$\begin{array}{ll}R_{f} & \text { Fluorescence ratio } \\ S c & \text { Schmidt number } \\ t & \text { time } \\ T & \text { Absolute temperature } \\ T_{i} & \text { Injection temperature } \\ U_{S} & \text { Maximum surface velocity } \\ V_{i} & \text { Injection velocity } \\ V_{c} & \text { Probe volume } \\ Y_{F} & \text { Fuel vapor mass fraction } \\ \text { Greek } & \\ \lambda & \text { Wavelength or heat conductivity } \\ \nu & \text { Stoichiometric coefficient (mass of fuel per mass of oxygen) } \\ \psi & \text { Stream function } \\ \rho & \text { Specific density }\end{array}$

\section{Subscripts}

*

$S$

$\infty$

$e q$

$c$

$a$

$l$

$g$

$i$

ref

ox
Normalized quantities

Droplet surface

Ambient conditions (far away from the flame front, in quiescent air)

Equilibrium

Droplet core

Ambient temperature in the flame

Liquid phase

Gas phase

Injection

Reference conditions according to the " $1 / 3$ " rule

Oxidizer

\section{REFERENCES}

Abramzon, B. and Sirignano, W.A. (1989) Droplet vaporization model for spray combustion calculations. Int. J. Heat Mass Transfer, 32(9), 1605.

Castanet, G., Lavieille, P., Lebouché, M., and Lemoine, F. (2003) Measurement of the temperature distribution within monodisperse combusting droplets in linear stream using two colors laser-induced fluorescence. Exp. in Fluids, 35, 563.

Chiang, C.H., Raju, M.S., and Sirignano, W.A. (1992) Numerical analysis of convecting, vaporizing, fuel droplet with variable properties. Int. J. Heat Mass Transfer, 35, 1307. 
Chiang, C.H. and Sirignano, W.A. (1993) Axisymmetric calculation of threedroplets interactions. Atomization Sprays, 3, 91.

Copetta, J. and Rogers, C. (1998) Dual emission laser induced fluorescence for direct planar scalar behavior measurements. Exp. in Fluids, 25, 1.

Gutheil, E. (1995) Numerical analysis of the autoignition of methanol, ethanol, n-heptane and n-octane sprays with detailed chemistry. Comb. Sci. Tech., 105, 265.

Jin, J.D. and Borman, G.L. (1985) A model for multicomponent vaporization at high ambient pressures. Combust. Emis. Anal., P-162, 213.

Lavieille, P., Lemoine, F., Lavergne, G., Virepinte, J.F., and Lebouché, M. (2000) Temperature measurements on droplets in monodisperse stream using laserinduced fluorescence. Exp. in Fluids, 29, 429.

Lavieille, P., Lemoine, F., Lavergne, G., and Lebouché, M. (2001a) Evaporating and combusting droplet temperature measurements using two colors laserinduced fluorescence. Exp. in Fluids, 31, 45.

Lavieille, P., Lemoine, F., and Lebouché, M. (2001b) Combusting droplets temperature measurements using two colors laser-induced fluorescence: preliminary results and perspectives. C.R. Acad. Sci. Paris, Série II-b, t. 329, 557.

Lavieille, P., Lemoine, F., and Lebouché, M. (2002) Experimental investigation on interacting low evaporating droplets temperature in linear stream using two colors laser induced fluorescence. Comb. Sci. Tech., 174, 117.

Lemoine, F., Antoiner, Y., Wolff, M., Lebouché, M. (1999) Simultaneous temperature and 2D velocity measurements in aturbulent heated jet using combined laser-induced fluorescence and LDA, Exp. in Fluids, 26, 315.

Mulholland, J.A., Srivastava, R.K., and. Wendt, J.O.L. (1988) Influence of droplet spacing on drag coefficient in non-evaporating, monodisperse stream. AIAA J., 26(10), 1231.

Renksizbulut, M. and Yuen, M.C. (1983) Numerical study of droplet evaporation in a high temperature stream. J. Heat Transfer, 105, 389.

Sazhin, S., Dombrovsky, L.A., Krutitski, P., Sazhina, E.M., and Heikal, M.R. (2002) Analytical and numerical modelling of convective and radiative heating of fuel droplets in diesel engines. Proceedings of the International Heat Transfer Conference, 18-23 August, Grenoble, France (proceeding on CD).

Silverman, M.A. and Dunn-Rankin, D. (1994) Experimental investigation of a rectilinear droplet stream flame. Comb. Sci. Tech., 100, 57.

Sirignano, W.A. (1999) Fluid Dynamics and Thermal Transport of Droplets and Sprays, Cambridge University Press Cambridge.

Talley, D.G. and Yao, S.C. (1986) A Semi-empirical approach to thermal and composition transients inside vaporizing fuel droplets, Proc. Combust. Instit., 21, 609.

Virepinte, J.F., Biscos, Y., Lavergne, G., Magre, P., and Collin, G. (2000) A rectilinear droplet stream in combustion: droplet and gas phase properties. Comb. Sci. Tech., 150, 143. 